\title{
Sistema de Información Para Evento Deportivo: Caso de Aplicación Básquetbol
}

\author{
Arnulfo Castro, Saúl González, Carlos A. Ochoa, Lidia H. Rascón \\ Universidad Autónoma de Ciudad Juárez, Instituto de Ingeniería y Tecnología, Av. del Charro 450 \\ Norte, 32310, Ciudad Juárez, Chihuahua-México (e-mail: arncastr@uacj.mx; saugonza@uacj.mx; \\ megamax8@hotmail.com; Irascon@uacj.mx)
}

Recibido Dic. 01, 2009; Aceptado Feb. 09, 2010; Versión Final recibida Abr. 22, 2010

\begin{abstract}
Resumen
El artículo describe el desarrollo de una aplicación Web dinámica con una Base de Datos y la capacidad de administrar datos registrados en un torneo de Básquetbol. Algunas de las facilidades que proporciona la aplicación incluyen la capacidad de registrar acciones que suceden en un encuentro tales como: faltas, robos de balón, cambios, y cantidad de encestes por jugador. Así mismo se tiene capacidad de registrar los datos de cada persona que participa en el equipo, tales como entrenador, asistente, y jugadores entre otros: Además se puede registrar información general del torneo tales como: árbitros, encuentros, y características de las instalaciones entre lo más destacable.
\end{abstract}

Palabras clave: sistema de información, bases de datos, diseño de sistemas, aplicación Web

\section{Sports Information System: An Application case in Basketball}

\begin{abstract}
This work presents the development of a dynamic Web application and a database, both suited to manage data of a basketball tournament. Some features available in this system include the registering of actions occurring during a match, such as fouls, steals, substitutions, and scoring by player, etc. Also, it is possible to register data about people participating in the teams, such as coaches, assistants, and players among others. Additionally, the system can register general information of the tournament such as schedule of matches, courts, and facilities, among others.
\end{abstract}

Keywords: information system, databases, systems design, web application 


\section{NTRODUCCIÓN}

El presente documento describe el desarrollo de una aplicación que tiene como objetivo principal facilitar el manejo de información de uno o varios torneos del deporte básquetbol a través de un sistema de información, el cual tiene las características de comportamiento de un sistema que de acuerdo con Reyes et al. (2007) es el conjunto de elementos dinámicamente interrelacionados que tienen un propósito determinado. De esta definición se desprende una implicación básica que tiene relación con el manejo de los datos; por lo tanto, la influencia mutua entre sus componentes repercute en cualquiera de sus elementos y afecta invariablemente al resto del sistema de información.

Cuando se implementa un sistema de información se debe determinar el universo del discurso (UdD) en el cual se maneja la mayor cantidad de información con el objetivo de que ésta pueda ser utilizada en análisis estadístico o en la aplicación de herramientas de la Minería de Datos. En este caso el universo del discurso es uno o varios torneos de básquetbol.

De acuerdo con Bertalanffy (1999) el aplicar elementos de análisis de datos implica que: "la importancia de reconocer las propiedades dinámicas de los sistemas es que éstas constituyen parámetros básicos de patrones que luego sustentan la aplicación de modelos para describir o concebir nuevos sistemas". Partiendo de esta aseveración se realizó el análisis y el desarrollo de un sistema de información, el cual incluye múltiples aspectos a considerar. Dentro de los de mayor importancia se tienen: el torneo, el encuentro y los participantes del torneo; a su vez se establece cómo se interrelacionan para administrar la información a través de una aplicación Web.

El desarrollo del sistema de información se dividió en dos secciones: la primera, el análisis y desarrollo de la Base de Datos (BD); la segunda, el análisis y desarrollo de la aplicación que accede a los datos, administrados por la base de datos.

La primera sección está formada por dos sub secciones, estas se refieren al análisis y desarrollo de la BD. Implementar la BD es necesario para controlar los datos de acuerdo a las características de un torneo y para satisfacer las necesidades de cada uno de los miembros que participa en dicho torneo de éste deporte, de tal manera que se pueden manejar aspectos que hacen referencia a las actividades de uno o varios torneos, incluyendo el control de usuarios, eventos en encuentros incluyendo algunas facilidades para el manejo de estadísticas, así como videos de algunos aspectos y eventos previamente registrados.

La primera sub-sección maneja la obtención de requisitos (Elmary, 2002), los cuales se quieren manejar de manera computacional y se obtienen a partir del análisis de requerimientos que registra todos los supuestos necesarios a manejar del UdD por medio del sistema de información.

La segunda sub-sección modela los requerimientos mediante el modelo Entidad-Relación, el cual permite modelar de manera conceptual todos los aspectos del UdD; algunos de los elementos que se presentan son: el esquema conceptual representado a través del Modelo Entidad-Relación, al cual se le aplica el proceso de conversión y se obtiene el esquema relacional de la base de datos del UdD que tiene la capacidad de manejar toda la información de los torneos.

La segunda sección refiere al análisis, desarrollo e implementación de la aplicación Web que accede a los datos contenidos en la BD previamente desarrollada, la aplicación Web es el intermediario entre los usuarios y el sistema de administración de los datos. Ambas aplicaciones interaccionan entre sí para dar respuesta a las diferentes necesidades de información de los diversos tipos de usuarios que pueden hacer uso del sistema que controla y administra el torneo de básquetbol.

El desarrollo de las aplicaciones Web con accesos a bases de datos no es nuevo (Elmasri, 2002), existen múltiples aplicaciones que utilizan esta forma de administrar los datos y que se asemejan a los sistemas de información tradicionales sólo que utilizando una tecnología diferente. De igual forma se tienen que considerar para su desarrollo aspectos que inciden directamente sobre el ciclo de construcción de la aplicación. 
Dentro del desarrollo de esta aplicación se hizo una división, una primera parte incluye aspectos que tienen que ver con: análisis de factibilidad, obtención de requerimientos, análisis y diseño de la aplicación haciendo uso de los datos contenidos en la base de datos, modelando la interacción dinámica de los datos además de modelar la manera de registrar los eventos en cada encuentro y en cada aspecto relacionado con el básquetbol.

La segunda parte consiste en la implementación, validación, prueba y mantenimiento; La aplicación Web es la parte fundamental a través de la cual los usuarios acceden al sistema de información y pueden realizar acciones tales como registrar torneos, encuentros, acciones de un encuentro y generar los distintos tipos de reportes y consultas sobre los datos que se registran.

Existen muchas aplicaciones de Sistemas de Información, López (2009) plantea el desarrollo de un sistema de información aplicado a la cultura, de igual forma Cruz (2005) plantea la utilización de sistemas de información geográficos territoriales; por otro lado, Navarrete y Banqueri (2008) analiza las capacidades de los sistemas de información en la biomedicina, Braga (2003) utiliza un sistema de información para administrar el conocimiento a través de una aplicación web.

\section{DESARROLLO DE LA BASE DE DATOS}

Durante las etapas del diseño de una BD se deben cumplir ciertas etapas las cuales forman parte de la metodología del buen diseño, las cuales según Adoración et al. (2007) son: obtención de requisitos, análisis de restricciones, diseño conceptual, elección del Sistema Gestor de Base de Datos (SGDB), transformación del esquema de datos, diseño físico, implementación y ajuste del sistema de base de datos. Tomando como base estos elementos se presentan los principales factores que se tomaron en consideración para el diseño de la base de datos utilizada en esta aplicación.

\section{Obtención y análisis de requisitos}

El primer objetivo es comprender el UdD que se va a modelar, en este caso un juego de básquetbol. Esto incluye registrar y analizar los requerimientos y reglas que se deben cumplir y que forman parte de este deporte. La documentación de los requerimientos de los usuarios es fundamental, de manera que cuando se obtiene esta información se inicia el proceso de análisis de la misma.

Algunos de los aspectos que se consideraron son los siguientes: Identificación de los principales usuarios del sistema y las acciones que realizarán dentro de la BD. Documentar el análisis de las reglas del deporte considerando acciones en un encuentro y fuera del mismo, tales como las reglas que fundamentan un torneo al que pertenece dicho encuentro. Registro del uso que se le dará a la información por parte de los usuarios como: frecuencia de acceso, flujo de datos e interpretación de éstos para generar la información, vistas y tipos de accesos.

Algunos de los principales aspectos a considerar en un encuentro de básquetbol son las reglas oficiales de básquetbol (FIBA, 2005), clasificados según la persona o elemento involucrado, se presentan en la tabla 1.

\section{Esquema Conceptual Entidad Relación}

Para registrar los distintos datos de interés en la base de datos se debe considerar la aplicación del modelo Entidad-Relación (Elmasri et al., 2002) para obtener el esquema que represente el universo del discurso, obteniendo así el esquema lógico que se transformará en la base de datos. Así mismo, se obtienen las cardinalidades de cada relación entre las diversas entidades que forman parte del esquema lógico. Este proceso generó los elementos que se presentan en la tabla 2 donde las entidades, incluyendo las fuertes y las que dependen de estas, se registran en la columna izquierda. En la columna derecha se presentan las relaciones que se registraron entre las diversas entidades que forman el UdD Básquetbol. 
Tabla 1: Restricciones del deporte Básquetbol

\begin{tabular}{|c|c|}
\hline $\begin{array}{l}\text { Persona o } \\
\text { elemento }\end{array}$ & Actividad o regla a cumplir \\
\hline \multirow{4}{*}{ Jugador } & No puede forma parte de dos equipos en el mismo torneo \\
\hline & Debe de cumplir con la edad que define la cateqoría del torneo \\
\hline & Debe de cumplir con el sexo que define la cateqoría del torneo \\
\hline & No Puede ser asistente, entrenador o árbitro al mismo tiempo \\
\hline \multirow{4}{*}{ Equipo } & Deberá contar con un mínimo de 8 v Máximo de 12 juqadores \\
\hline & Deberá de contar con un entrenador v un asistente \\
\hline & no puede participar en dos torneos \\
\hline & no puede tener jugadores de ambos sexos \\
\hline \multirow{4}{*}{ Entrenador } & La edad deberá de ser mavor de 20 v menor o iqual a 90 años \\
\hline & no puede entrenar a dos equipos en el mismo torneo \\
\hline & no puede ser iuaador o asistente o arbitro al mismo tiempo \\
\hline & Deberá permanecer en la banca y no podrá entrar a la cancha \\
\hline \multirow[t]{3}{*}{ Asistente } & La edad deberá ser mavor de $16 \mathrm{v}$ menor o iqual a 90 años \\
\hline & no puede forma parte de dos equipos en el mismo torneo \\
\hline & Deberá permanecer en la banca y no podrá entrar a la cancha \\
\hline \multirow{7}{*}{ Tiempo } & Un juego dura 40 minutos \\
\hline & $\begin{array}{l}\text { Cada encuentro deberá ser dividido en } 4 \text { periodos o cuartos de } 10 \text { minutos cada } \\
\text { Uno }\end{array}$ \\
\hline & Entre cada inicio del 2 v 4 periodo hav un tiempo de 2 minutos de descanso \\
\hline & Antes del inicio del 3 periodo, existe un tiempo de descanso de 10 minutos \\
\hline & $\begin{array}{l}\text { se detiene cada vez que ocurre alguno de los siguientes eventos: faltas, tiros } \\
\text { libres, saques, tiempos fuera }\end{array}$ \\
\hline & $\begin{array}{l}\text { El equipo atacante tendrá un tiempo de } 8 \text { segundos para trasladar el balón hacia } \\
\text { la media chancha del rival }\end{array}$ \\
\hline & $\begin{array}{l}\text { El equipo atacante tendrá un tiempo de } 24 \text { segundos, incluyendo los } 8 \text { segundos } \\
\text { anteriores, para realizar un tiro en la canasta y este a su vez haga contacto con } \\
\text { el aro }\end{array}$ \\
\hline \multirow[t]{2}{*}{ Partido } & $\begin{array}{l}\text { Dentro del área un jugador o mas no pueden permanecer más de } 3 \text { segundos } \\
\text { dentro del área, esta regla aplica únicamente para el equipo que se encuentra } \\
\text { en ese momento al ataque }\end{array}$ \\
\hline & Cada partido será dirigido por dos árbitros \\
\hline
\end{tabular}

Una vez que se han considerado todas las restricciones se procede a realizar el Esquema EntidadRelación (E-R) donde se reflejan todas las características obtenidas de los requerimientos de usuario incluyendo la forma en como se relacionan las entidades y las restricciones entre estas. El resultado se presenta en la figura 1, la cual muestra un esquema lógico del diseño de la BD del sistema de información básquetbol.

Una vez realizado el esquema E-R se procede a la conversión del mismo a esquema relacional (Adoración, 2007) y como resultado de éste proceso se obtiene la representación del UdD en esquema relacional como se puede apreciar en la figura 2, la cual presenta las principales entidades y como se interrelacionan entre ellas, indicando el tipo de relación y la cardinalidad que deben mantener las distintas relaciones. 
Tabla 2: Entidades y relaciones obtenidas en el análisis del UdD del Básquetbol

\begin{tabular}{|l|l|}
\hline Entidades Fuertes y Débiles & Relaciones \\
\hline Persona & AccionParti \\
\hline Categoria_Torneo & ArbxParti \\
\hline Institucion & RobxAcc \\
\hline Violacion & InstaxParti \\
\hline Robo & TabxAcc \\
\hline Cambio & LesxAcc \\
\hline Falta & CamxAcc \\
\hline Enceste & Tfuera \\
\hline Videos & JugxAcc \\
\hline ClasifLesion & EntXAcc \\
\hline Jugador & FalxAcc \\
\hline Entrenador & VioxAcc \\
\hline Partido & \\
\hline Equipo & \\
\hline Instalacion & \\
\hline Usuarios & \\
\hline Torneo & \\
\hline Asistente & \\
\hline Arbitro & \\
\hline
\end{tabular}

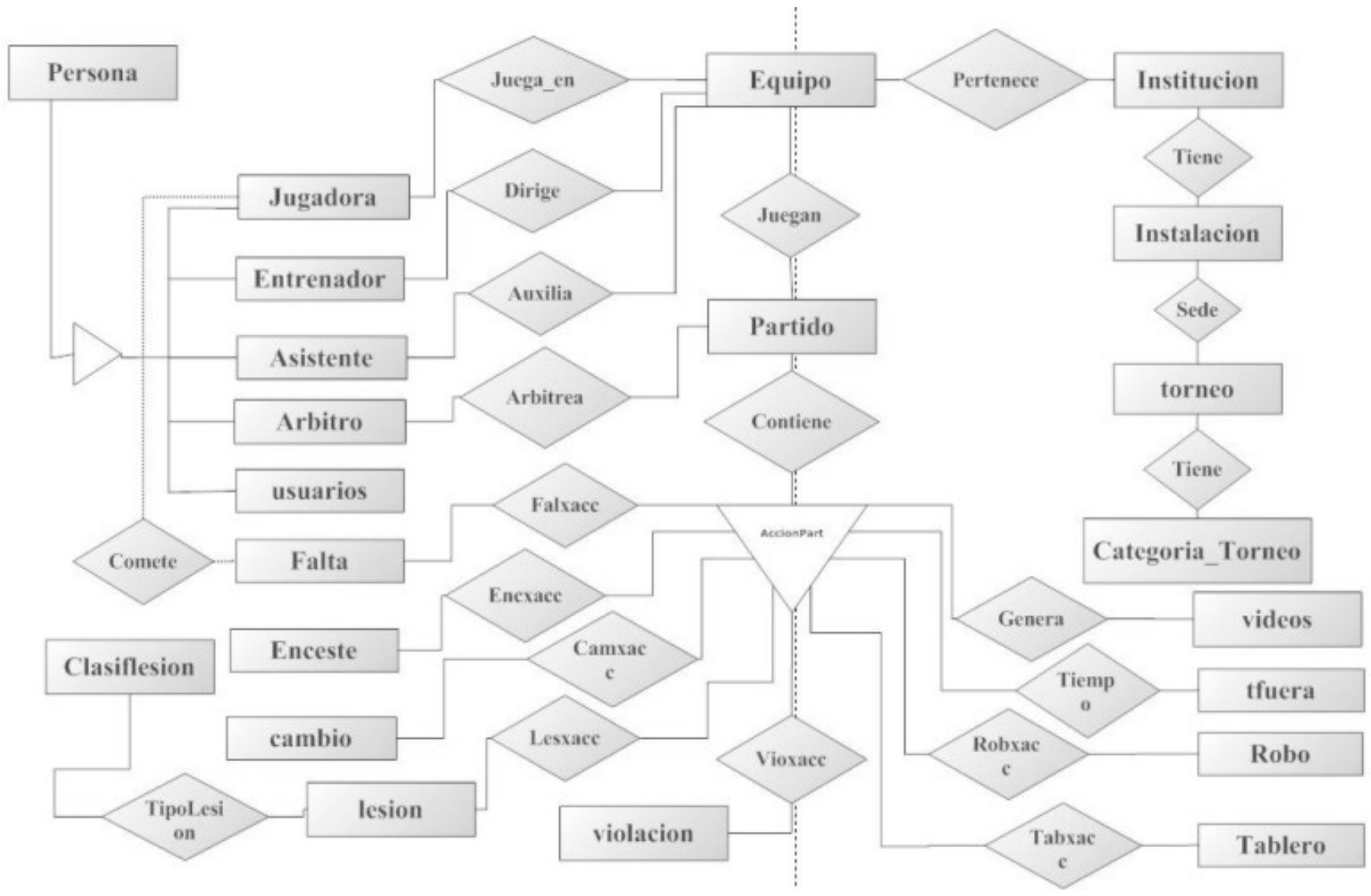

Fig. 1: Esquema lógico E-R del UdD básquetbol 


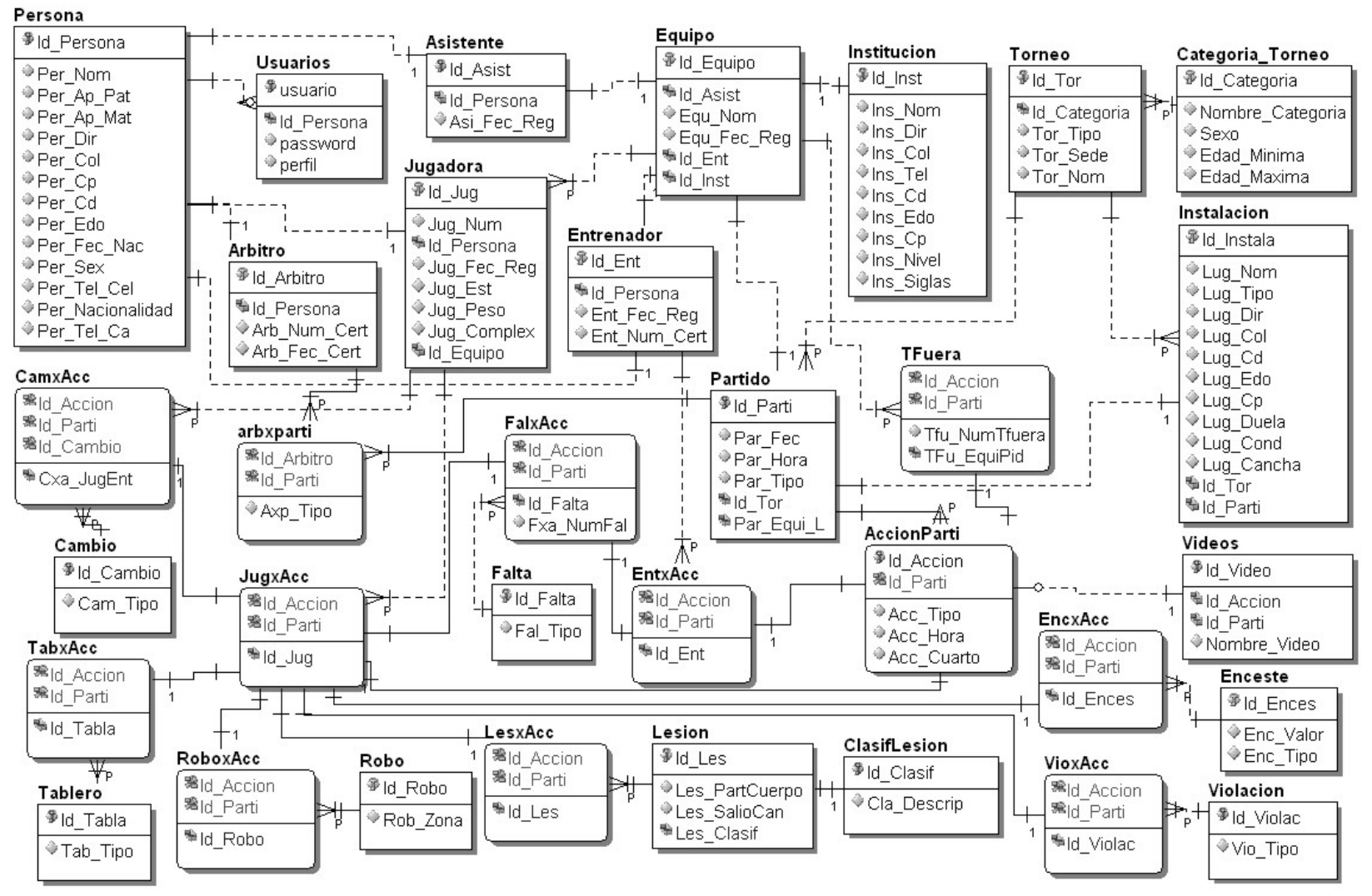

Fig. 2: Esquema Relacional parcial del UdD Básquetbol

Este esquema relacional presenta aspectos tales como: las reglas de integridad referencial marcando las claves primarias, foráneas y cardinalidades (Ramez, 2005). Es importante resaltar que se incluyen las tablas que representan las diferentes acciones de cada partido, tales como: robo, tablereo y lesiones, por mencionar algunas. Una vez que se tiene este esquema relacional se convierte a esquema físico el cual se implementa en el SGBD elegido, incluyendo las restricciones del UdD que no se lograron representar en cada esquema presentado hasta esta parte del proceso y que forman parte de los requerimientos, obteniendo así la construcción final de la base de datos.

\section{DESARROLLO DE LA APLICACIÓN WEB}

El diseño conceptual de ésta aplicación Web incluye varios elementos que se consideraron, algunos de los más importantes son: control de usuarios, diseño de rutas de datos también conocido como diagramas de flujos de datos y diagramas de navegación entre la aplicación Web y la BD que soporta todos los datos que intervienen en el sistema. Los datos se transforman en información durante este proceso gracias a la interpretación que le dan los usuarios.

\section{Diseño de la Aplicación}

Para el diseño de la aplicación se tomaron en cuenta los siguientes elementos: requerimientos de acceso de los usuarios (Hairong, 2008), restricciones del deporte, páginas específicas de cada usuario, datos necesarios para cada página, flujos de datos de la base de datos a la página, acciones de navegación sobre las páginas.

Una vez registrados y modelados estos elementos, se procede a construir el modelo arquitectónico que representa la aplicación y el cual controla todos los datos que tienen relación con el sistema de información, y es utilizado por todas las páginas que formarán el sitio web.

Algunos de los elementos que se han modelado se presentan en la figura 3, la cual esta divida en 2 
partes y presenta el proceso de entrada a la aplicación web, en la primera parte (a) se presenta el proceso para registrarse el cual determina el tipo de usuario además de su identificación. La segunda parte presenta dos sub-secciones figura 3 (b) de las quince que se implementaron para controlar la información que se maneja del UdD a las cuales se pueden aplicar las acciones necesarias de inserción, actualización, eliminación, etc.



Fig.3a: Diagrama a bloques de Entrada

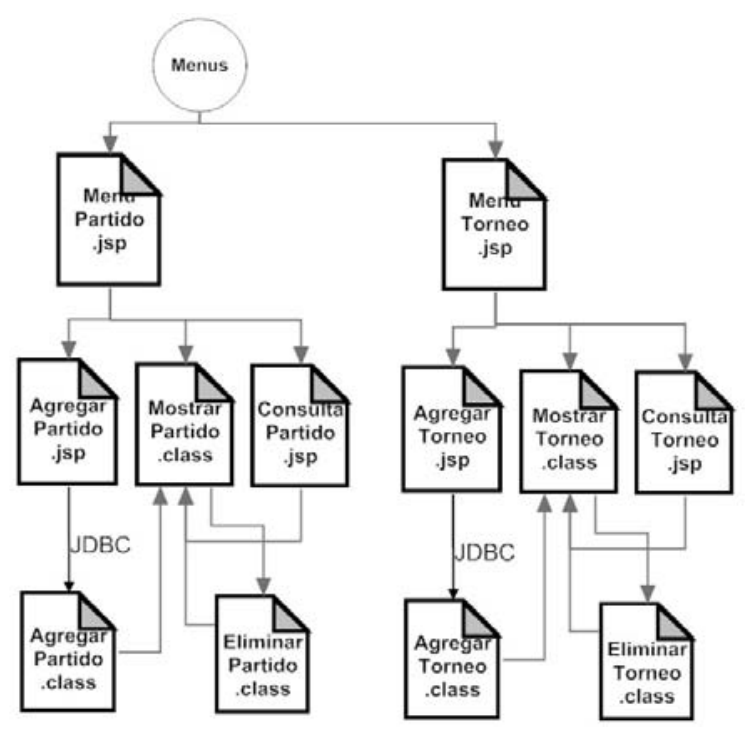

Fig. 3b: Diagrama a bloques de Menús

Una parte del desarrollo de la aplicación implica el crear las restricciones de uso de acuerdo a cada perfil y se limitan las acciones de los mismos de acuerdo con lo establecido en las restricciones originales recogidas al inicio del diseño del sistema, de tal forma que se limitan las acciones que le son permitidas a los distintos tipos de perfiles de usuarios que se pueden tener en la aplicación se crearon tres perfiles administrador, organizador y entrenador.

Cuando un usuario se identifica y accede a el área de administración de datos éste se registra dentro de la aplicación, puede modificar a través de la aplicación los datos almacenados en la base de datos, el tipo de modificaciones que puede realizar dependen del tipo de perfil, si se identifica como administrador puede modificar cualquier dato de: usuarios, torneos, jugadores, equipos, etc. Puede modificar información de treinta y tres tablas que conforman la base de datos entre las cuales se encuentran tablas básicas y complementarias además de las consultas de tipo reportes que se pueden realizar desde la página de inicio.

\section{La interfaz de la aplicación}

Al momento de invocar el sitio se presenta una pantalla de visualización de tipo genérica la cual permite hacer consultas estadísticas, fichas técnicas, videos de acciones, etc. Cuando un usuario desea acceder al sitio siempre debe ser mediante una identificación, el sistema presenta una validación de entrada donde se solicitan tres datos: usuario, contraseña y tipo de usuario, tal como se muestra en la figura 4. Cuando el usuario proporciona los datos de acceso se inicia un proceso de validación en el que el sistema identifica inequívocamente el tipo de usuario y responde de acuerdo al perfil del mismo. Si la validación es favorable se establece la respuesta de acuerdo a ese perfil.

Cuando se tienen los perfiles pre-determinados (Oros, 2004) y las acciones que éstos pueden desarrollar dentro del sistema, el paso siguiente es mostrar las páginas que utilizarán. Se parte del perfil del administrador, entrenador o jugador, en este caso se presenta el perfil más complejo, el del administrador ya que este tiene acceso a la configuración del sitio y puede administrar cualquier dato del sistema.

Control de Usuarios. Se crearon perfiles de acceso, los cuales determinan las acciones permitidas a cada usuario sobre los datos almacenados en la BD (Elmasri, 2002). Así mismo, éste perfil determina 
el tipo de respuesta del servidor al usuario que accede al sistema. La administración de los perfiles forma parte de la misma BD, quedando restringido su uso al administrador del sitio el cual puede determinar la asignación de perfiles de usuarios incluyendo los posibles cambios de tipo de perfil, en la figura 5 se muestra la pantalla que utiliza el administrador para registrar los datos de una persona a la que se le pueden asignar los perfiles mencionados.

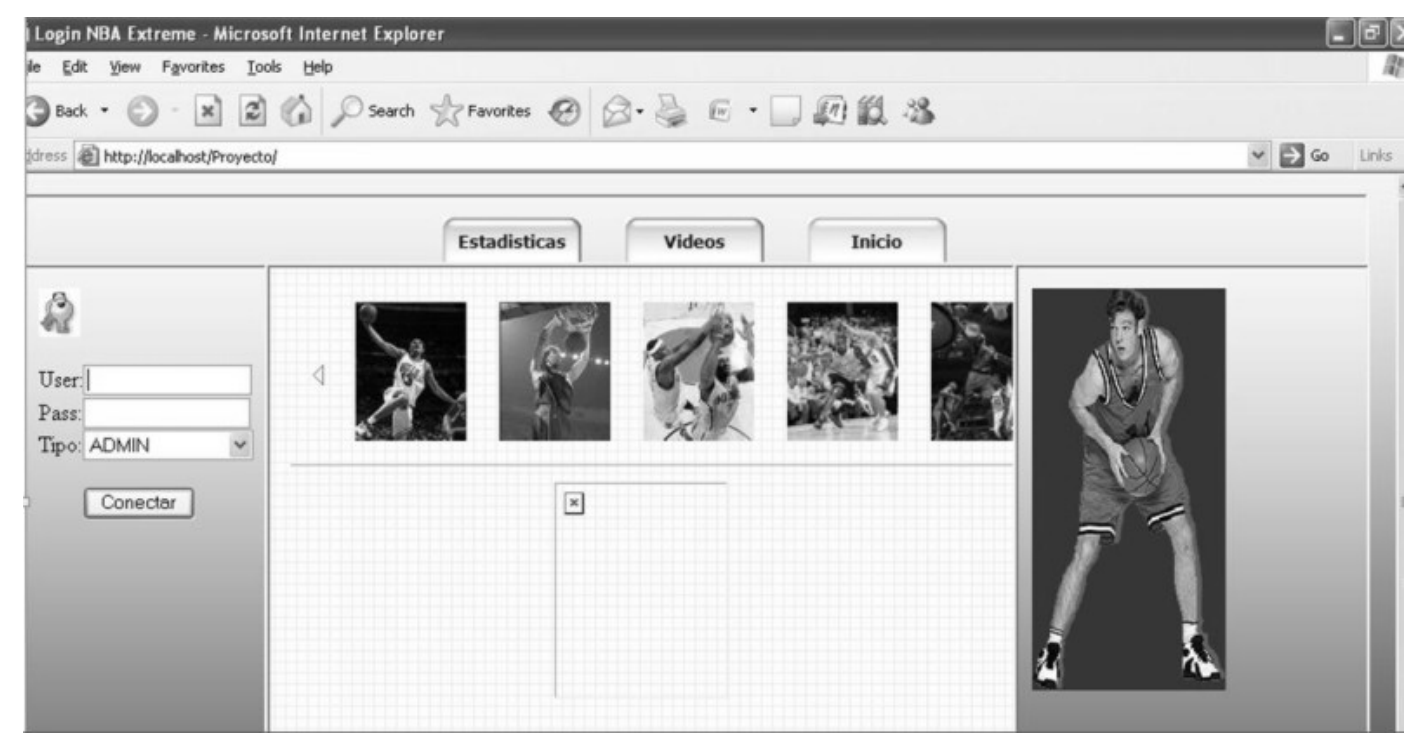

Fig. 4: Pantalla de validación de usuario

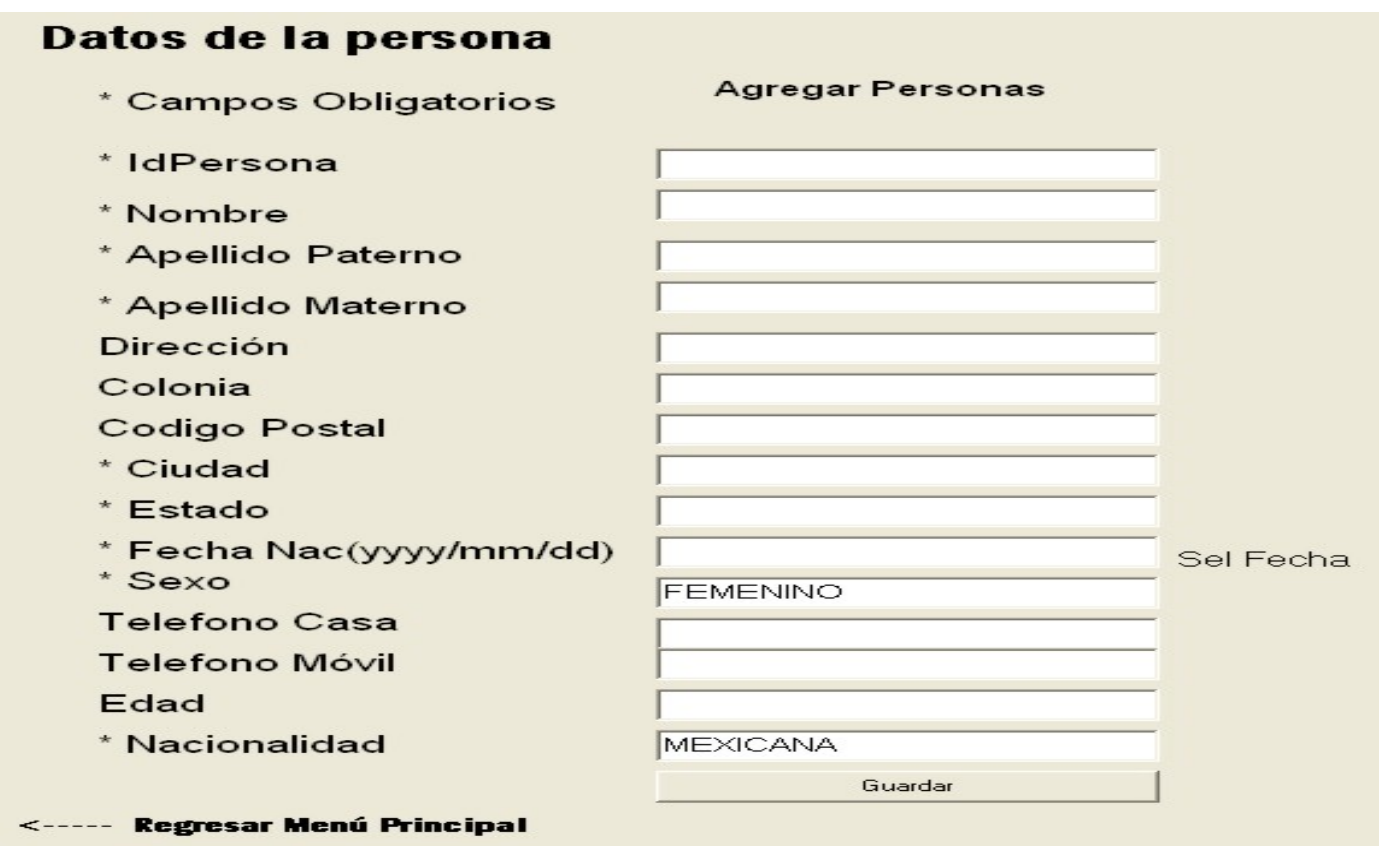

Fig. 5 Registro de persona perfil administrador

Cuando se accede como usuario entrenador, este puede administrar todos los elementos que se relacionan con su equipo y las acciones de cada encuentro. De igual forma puede modificar cualquier dato referente a los miembros de su equipo y generar cualquier reporte de algún encuentro específico o de todos los encuentros registrados, ya sea independientes entre si o como parte de un torneo. Los reportes han sido previamente configurados para ser obtenidos de la información que se deposita en la BD.

El usuario normal permite acceder a diversas opciones así como distintos tipos de reportes. Permite realizar búsquedas sobre datos específicos de jugadores y la visualización de los videos referentes a 
las acciones ocurridas en algún encuentro en particular pero no permite hacer modificaciones sobre los datos que obtienen o visualizan.

Cada uno de los eventos de un encuentro pueden ser registrados a través de esta aplicación, este proceso puede ser realizado por el tipo específico de usuario al que le corresponde y dependerá de los privilegios que tenga. Así, un entrenador puede registrar una acción dentro de un encuentro utilizando la opción "Registro de Acción" la cual corresponde a una opción específica que será presentada de acuerdo a el perfil de usuario y donde se puede elegir a que usuario se asignará cada evento a registrar dentro de un encuentro cotidiano. Además del registro de la acción, esta se puede enlazar con el momento exacto en el que ocurrió dentro del encuentro; esto se consigue registrando el tiempo exacto en el que ocurrió en el video del partido (previamente tomado) y con este dato la aplicación puede obtener una sección del video original y presentarlo al usuario cuando este lo solicita en la página Web.

\section{RESULTADOS}

Se obtuvo una base de datos que es capaz de registrar cada uno de los eventos que ocurren dentro de un torneo o torneos de básquetbol, incluyendo partidos y eventos dentro de los mismos tales como faltas, encestes, y cambios, por mencionar los más importantes.

Se obtuvo una aplicación que hace uso de la base de datos antes mencionada y que permite a través de la web administrar y manejar toda la información relevante de un torneo o torneos del deporte del básquetbol, incluyendo la capacidad de relacionarlos con el video correspondiente a dicho partido.

Se obtuvieron más de treinta tablas que reflejan todos los aspectos que se plantearon al inicio del desarrollo de la base de datos incluyendo las relaciones entre cada una de estas tablas, así como las reglas de integridad que deben guardar los datos dentro de la misma base de datos.

Se Implementó una base de datos que es capaz de soportar la aplicación de tecnologías de minería de datos ya que el dominio de los datos permite realizar consultas sobre datos numéricos en la gran mayoría.

Es posible manejar la administración de usuarios y la consecuente jerarquización de los mismos, gestionándolo a través del mismo sitio Web, lo cual genera diversas acciones para cada tipo de usuario dentro del sistema.

\section{CONCLUSIONES}

Es posible generar sistemas con capacidades de apoyo a usuarios en más de un sentido, ya que el desarrollo de la tecnología permite mayores opciones para generar mejores soluciones. Un sistema con las capacidades antes mencionadas es una buena opción para ser evaluada en instituciones deportivas ya que permite una mayor eficiencia en el manejo de la información del deporte.

De manera similar, es posible adaptar los esquemas de modelado de base de datos a las necesidades propias de casi cualquier problema de administración y control de datos.

Con la implementación del sistema de Información para el deporte básquetbol se da respuesta a una de las necesidades imperiosas para manejo de éste deporte a través de un medio digital puede administrar de manera más eficiente la información y generar cualquier tipo de reportes para administrar cualquier cantidad de torneos y partidos por parte de los usuarios como el entrenador 0 jugadores.

Se cumplió con el objetivo inicial, el cual pretendía administrar la información de un deporte aplicando cada uno de los elementos básicos de modelado en el Sistema de Información. 
Se logró crear un sistema con capacidades superiores a las básicas y con una posible interacción de la tecnología de la minería de datos, ya que permite analizar muchos de los aspectos que no se registran cotidianamente en los encuentros de un torneo.

\section{REFERENCIAS}

Adoración M., P. Martínez, E. Castro, J.M. Cavero, D. Cuadra, A.M. Iglesias, C. Nieto; Diseño de Bases de Datos: Problemas resueltos, $2^{a}$ ed., RA-MA, 490, España (2007)

Bertalanffy A.E.; Interdisciplinariedad en educación. Buenos Aires: Magisterio del Río de la Plata;1999. Disponible en:

http://www.virtual.unal.edu.co/cursos/sedes/manizales/4060001/Material_extra/Lect uras\%20Sugeridas/Interdisciplinariedad\%20en\%20educacion.doc;[Consultado: 4 de Junio del 2007]

Braga J.,C. Kimble, A. Rocha; Organisational Memory Information Systems An Example of a Group Memory System for the Management of Group Competencies; Journal of Universal Computer Science, vol. 9, no. 12 (2003)

Cruz R., E.G. Canino, S.V. Rodríguez, R. Muñoz; Organización de los proyectos de sistemas de información geográfica territoriales en Cuba; ISSN 1131-9100, No 102, 2005, pags. 18-23 disponible en: http://dialnet.unirioja.es/servlet/articulo?codigo=1173922 (2005)

Elmasri R.A., Navathe S.B.; Fundamentos de Sistemas de Base de Datos, $3^{\mathrm{a}}$ ed., Addison Wesley, 497-530, Madrid, (2002)

FIBA; Reglas oficiales de Básquetbol, http://www.fiba.com/ (2005)

Yu H., M. Davis, C.S. Wilson, F.T. Cole; Object-relational datamodelling for informetric databases, ELSERVIER, 240-251,(2008)

López R., F.J. Martínez, M. López; Diseño de un sistema de información web para la gestión cultural. Ibersid. 29-34. ISSN 1888-0967 (2009)

Navarrete J. y J. Banqueri; Los sistemas de información científica: herramientas para medir el impacto de la investigación biomédica; ISSN 0025-7753, Vol. 131, №. Extra 5, (2008)

Oros J.C.; Diseño de páginas Web interactivas con JavaScript y CSS., Alfaomega, 4a Ed., Ra-ma, 38-46, 55-56, 63-68, Madrid, España (2004)

Reyes L.M.; Consideraciones teóricas sobre los sistemas de información, los sistemas de información para la prensa y los sistemas integrados de información; EBSCO HOST, ACIMED; Vol. 15 Issue 1, p1, 20p, (2007) 